\title{
Investigation of the effect of cracks on the vibration processes in reinforced concrete structures
}

\author{
I. Shardakov, A. Shestakov, R. Tsvetkov, I. Glot \\ Institute of Continuous Media Mechanics, Ural Branch, Russian Academy of Science, 1, Korolev street, Perm, Russia \\ shardakov@icmm.ru,shap@icmm.ru,flower@icmm.ru,glot@icmm.ru
}

\begin{abstract}
The validity of the mathematical model describing the propagation of vibrations in the reinforced concrete structures (RC structures) was verified by comparing the experimental and numerical data. The proposed model allowed us to perform numerical experiments aimed at comparing vibrorecords obtained for the structure without defects and the structure with typical fracture caused by crack formation. Based on the results of comparison, an informative diagnostic parameter was proposed. This parameter makes it possible to control the nucleation and growth of cracks in a RC structure.
\end{abstract}

KEYwORDS. Vibration-based diagnostics; RC structure; Cracks; Full-scale model; Experiment; Simulation.

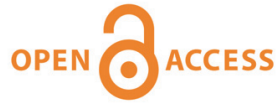

Citation: Shardakov I., Shestakov, A., Tsvetkov, R., Glot, I., Investigation of the effect of cracks on the vibration processes in RC structures, Frattura ed Integrità Strutturale, 46 (2018) 383-390.

Received: 12.08 .2018

Accepted: 22.09 .2018 Published: 01.10 .2018

Copyright: (C) 2018 This is an open access article under the terms of the CC-BY 4.0, which permits unrestricted use, distribution, and reproduction in any medium, provided the original author and source are credited.

\section{INTRODUCTION}

1 he results of studies of the behavior of complex building structures under critical conditions are important for solving the problems of their safe operation. One of the modern approaches that make it possible to solve problems of safe operation of structures is the use of automated deformation monitoring systems. The most important components of these systems are mathematical models that adequately describe the operation of the structure in subcritical and critical stages of its deformation.

The development and verification of such models is possible only when carrying out field experiments. For their implementation, a model structure was designed and assembled. This model reflects the deformation processes in fullscale engineering structure. It is a 4-storey monolithic reinforced concrete building on a scale of 1: 2 (Fig.1). Its total height is $6 \mathrm{~m}$, length is $9 \mathrm{~m}$, and width is $6 \mathrm{~m}$.

Automated deformation monitoring systems are based on the non-destructive control methods, in particular, the methods of vibration diagnostics $[1,2,3,4]$, among which one can differentiate between the methods analyzing natural vibrations $[5,6]$ and those examining the transient vibration processes [7, 8]. The vibration methods have found wide application because of the use of piesoceramic materials, which can operate both as actuators and sensors. They allow fault diagnosis in a broad frequency range and can be installed at the surface of the examined structure $[9,10]$ or embedded into it $[11$, 12].

The proposed approach is based on the registration of vibration processes in the structure under the action of impulse loads. It allows us to perform the local analysis of separate fragments of the structure while tracing the evolution of the 
front of shock waves as they pass through the structure. When the propagating wave front runs across defects, its frequency composition and propagation time change. By comparing the signals from the structure with and without defects, we can detect any faults, their location and size. The effectiveness of the solution of these problems depend on the selection of the place where the elastic wave is initiated, the spectral composition of the impulse and the place of recording the signal passing through the structure. A well-grounded choice of these parameters is possible in the framework of an appropriate mathematical model, which adequately describes the dynamic processes occurring in the structure.

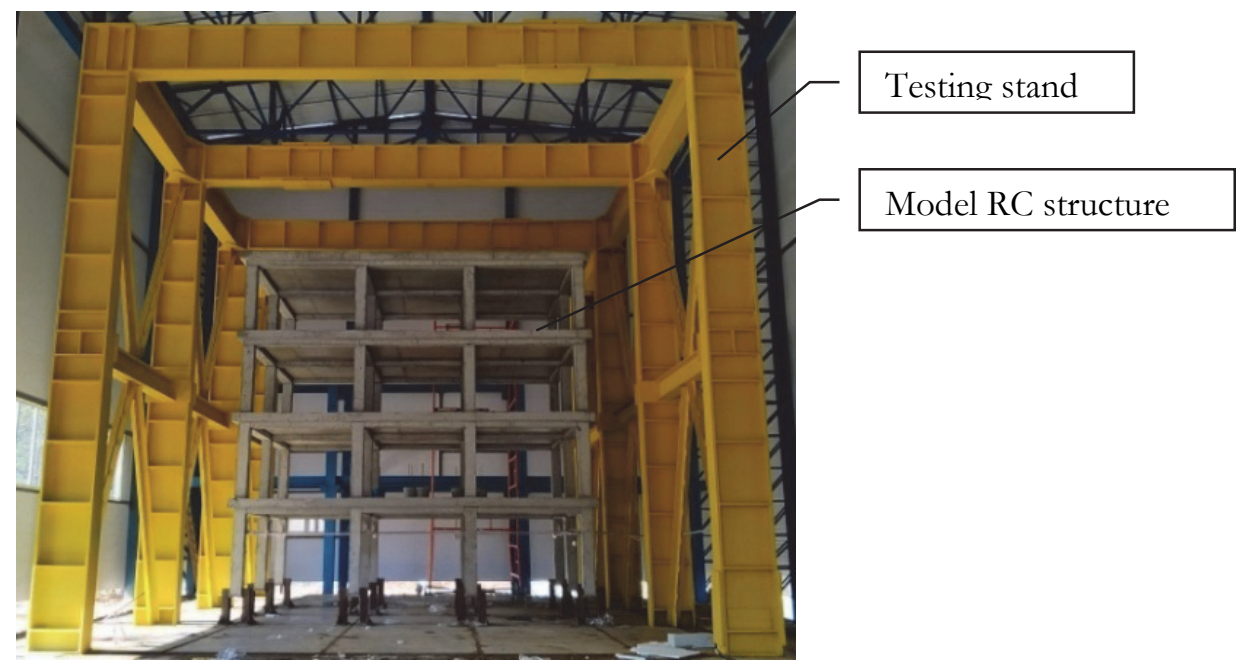

Figure 1: Testing stand with installed model RC structure.

In this study, the mathematical model describing the free vibrations of the structure caused by impact loading is verified. In the experiments, elastic waves in different elements of the model structure (columns, slabs) were excited by an impact of a $460 \mathrm{~g}$ striker. The resulting vibrograms were recorded by accelerometers mounted at different points of the structure. Registration of vibration processes in the model structure involved a synchronous recording of data from two accelerometers, one of which was located on the striker, and the other - at a certain point of the structure. The registration of the acceleration at the impact point made it possible to obtain the value of the impact force (as the product of the acceleration by the striker mass) as a function of time. These data were used to determine the force boundary condition at the point of impact necessary for solving the initial-boundary problem of propagation of a shock wave in a structure. This allowed us to compare the vibrograms obtained in the experiment and calculated on the basis of the proposed model.

This model was used as a framework for performing a series of numerical experiments, in which vibrograms obtained for the structure with crack formation at typical sites were compared with vibrograms for defect-free structure. Based on the results of these solutions we identified an informative diagnostic parameter, which reflects the processes of initiation and growth of cracks in reinforced concrete. This approach makes it possible to create an effective system for vibration control of the process of crack formation in RC structures.

\section{CONCEPTUAL DESCRIPTION OF THE MATHEMATICAL MODEL}

7 he object under study is a monolithic reinforced concrete building (Fig. 1), whose columns are mounted on metal supports. The dynamic behavior of the building is described in the framework of the linear theory of viscoelasticity. The concrete is assumed to be viscoelastic and isotropic, and steel reinforcement is modeled within the framework of the linear theory of elasticity. The mathematical model is represented by the following relations. Equations of motion:

$$
\operatorname{div} \sigma=\rho \frac{\partial^{2} \mathbf{U}}{\partial t^{2}}, \quad \mathbf{x} \in V
$$


Physical relations:

$$
\sigma=2 G\left(\frac{v}{1-2 v} I(\varepsilon) \mathrm{E}+\varepsilon\right)+2 \beta G\left(\frac{v}{1-2 v} I(\dot{\varepsilon}) \mathrm{E}+\dot{\varepsilon}\right)
$$

Geometrical relations:

$$
\left(\nabla \mathbf{U}+(\nabla \mathbf{U})^{\mathrm{T}}\right)
$$

Boundary conditions:

$$
\begin{aligned}
& \mathbf{n} \cdot \sigma=\mathbf{n} F(t), \quad F(t)=m W(t) / S_{\sigma}, \quad x \in S_{\sigma} \\
& \sigma \cdot \mathbf{n}=0, \quad \mathbf{x} \in S / S_{\sigma}
\end{aligned}
$$

Initial conditions for $t=0$ :

$$
U=0, \quad d U / d t=0
$$

Here: $V=V_{c} \cup V_{r}$ is the total volume of the structure, consisting of the volumes $V c$ and $V r$, occupied by concrete and steel reinforcement; $\sigma, \varepsilon, \dot{\varepsilon}$, E are, the tensors of stresses, strain, strain rates and a unit tensor, respectively; $I(\varepsilon), I(\dot{\varepsilon})$ are the first invariants of the strain tensor and of the strain rate tensor; $\mathbf{x}$ is radius vector of a point in the Cartesian coordinate system; $\mathbf{U}$ is the displacement vector; $\mathbf{n}$ is the unit vector oriented along the outward normal to the surface $S$ of the structure; $S_{\sigma}$ is the surface, to which an external impulse force is applied along its normal; $\varrho$ is the material density, $F(t)$ is a scalar that determines the time variation of the force impulse; $m$ is the striker mass, $W(t)$ (scalar) is the striker acceleration in the interval of striker contact with the surface of the structure (this value is recorded experimentally by an accelerometer mounted on the striker); $(\times, \cdot)$ denote vector and scalar products; $\nabla$ is the nabla operator; $G$ is the shear modulus, $v$ is the Poisson's ratio; $\beta$ is the parameter characterizing the dissipative properties of concrete. At $x \in V_{c}$ the values of $\varrho, G, v, \beta$ correspond to the physical properties of concrete, and at $x \in V_{r}$ they correspond to the physical properties of steel reinforcement.

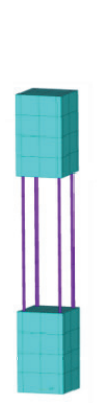

(a)

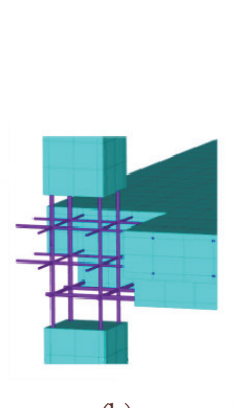

(b)

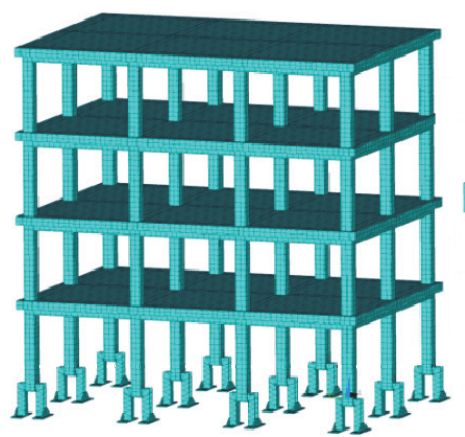

(c)

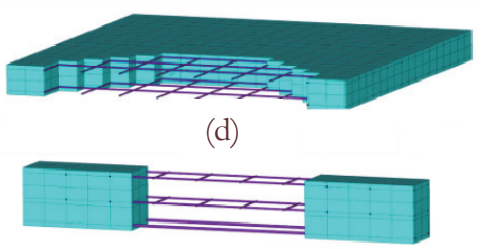

(e)

Figure 2: The finite element model of RC structure: (a) - a fragment of the column; (b) - reinforcement of the joint of the column, slab and cross beams; (c) - assembled structure; (d) - slab fragment; (e) - beam fragment.

The numerical implementation of the mathematical model is carried out by the finite element method using ANSYS software. Fig. 2 shows the finite element mesh of the model structure (the number of nodes is 506569). It represents the main components of the structure (columns, crossbars and slabs) and their reinforcement schemes.

The characteristic geometric parameters of the model structure and the physical properties of the materials are given below. 
- The main geometric characteristics of the model structure: column space is $2 \mathrm{~m}$; height of the floor is $1.5 \mathrm{~m}$; cross section of the column is $200 \times 200 \mathrm{~mm}$; cross-section of the cross beam is $200 \times 250 \mathrm{~mm}$; thickness of the slab is $150 \mathrm{~mm}$; diameter of the reinforcement of columns and lower chords of cross beams is $12 \mathrm{~mm}$, diameter of the other reinforcement is $8 \mathrm{~mm}$.

- Physical characteristics of concrete: $v=0.102 ; G=17.2 \mathrm{GPa} ; \beta=3.46 \cdot 10^{-7} ; \rho=2507 \mathrm{~kg} / \mathrm{m}^{3}$.

- Physical characteristics of steel: $v=0.3 ; G=76.9 \mathrm{GPa} ; \beta=0 ; \rho=7800 \mathrm{~kg} / \mathrm{m}^{3}$.

- Characteristic dimension of the finite element mesh: $0.1 \mathrm{~m}$.

- Integration time step: $10^{-5} \mathrm{~s}$.

\section{VERIFICATION OF THE MATHEMATICAL MODEL BASED ON EXPERIMENTAL RESULTS}

o verify the mathematical model, we performed a series of experiments, in which the dynamic deformation response of the structure to a locally applied external impulse force was analyzed. We used three loading schemes, of which two involved impact loading of the column and the registration of the deformation response to this impact at different points of the columns (Fig.3, a, b) and the third (Fig. 3, c) provided impact load of the lower edge of the floor slab and the registration of the response at its upper edge. Locations of the sensors recording the response of the structure to the externally applied impact loads are indicated by the red dots in the figures. These sensors recorded the vector component of acceleration directed along the normal to the surface, at which the sensors were located. Fig. 3 presents the fragments of the general model structure shown in Figs. 1 and 2c. These are the fragments in which the deformation response to the impact action was analyzed. The sizes of the selected fragments of the structure were chosen in such a way that at the points of recording the deformation responses there are no waves reflected from the boundaries of the fragment within the time interval under consideration. The external impulse force was generated by a striker with a mass of $460 \mathrm{~g}$. The value of acceleration $W(t)$, resulting from the impact of the striker on the surface of the structure, was recorded by an accelerometer ZetLab BC111 fixed to the striker. The range of recorded frequencies of this accelerometer is $0.5-15000 \mathrm{~Hz}$. The deformation response of the structure to the impulse force was registered by the ZetLab BC110 accelerometers, fixed at various points of the structure (the range of recorded frequencies is $0.5-10000 \mathrm{~Hz}$ ). Transformation of the collected sensor data to a digital form was performed synchronously using the ADC ZET 017-U8 with a frequency of $50 \mathrm{kHz}$.

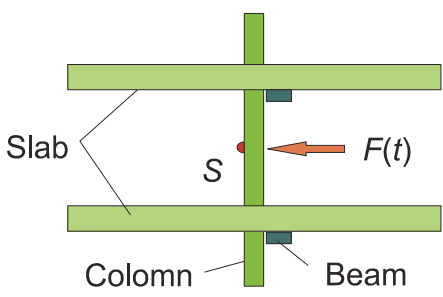

(a)

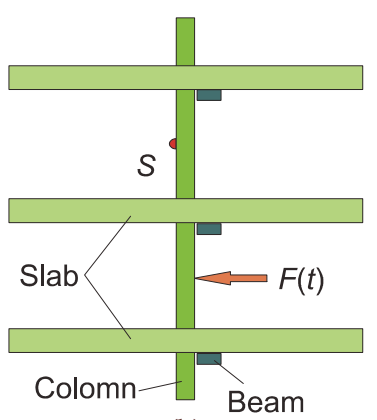

(b)

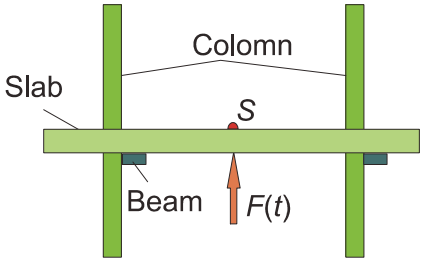

(c)

Figure 3: Schemes of loading and registration of response.

As an example, Fig. 4 shows the experimentally measured shape of the force impulse and the corresponding Fourier image (load diagram is given in Fig. 3a). As follows from these data, the impulse duration is $0.28 \mathrm{~ms}$, and the main part of impulse energy is localized in the frequency range of $0-5 \mathrm{kHz}$. It should be noted that the experimentally recorded value of the force impulse was used in the mathematical model (1) - (5) to specify the boundary condition (4). Then, the results of numerical simulation were compared with experimental data obtained from accelerometers recording the deformation responses to impact. When making a comparison, the calculated and experimental data were subjected to frequency filtering, ensuring the removal of the signal at frequencies above $6 \mathrm{kHz}$.

Fig. 5 shows a series of vibrograms and the corresponding Fourier images for three loading schemes (a, b, c) presented in Fig. 3. The experimental data are indicated by blue lines and the simulation data - by red lines. A comparison of the results obtained demonstrates good agreement between the model and the experiment in the frequency range from 0 to $6 \mathrm{kHz}$. 


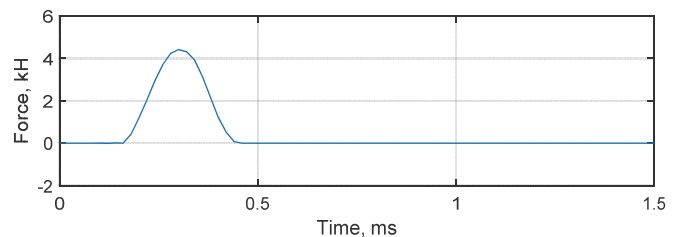

(a)

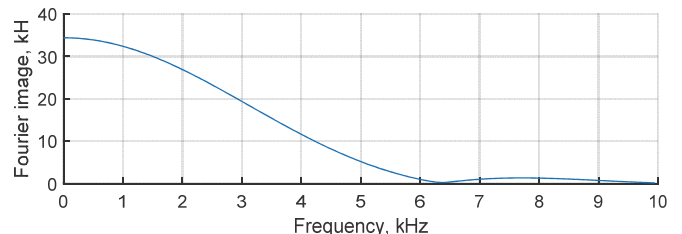

(b)

Figure 4: Impulse load: (a) - force evolution. (b) - Fourier transform.

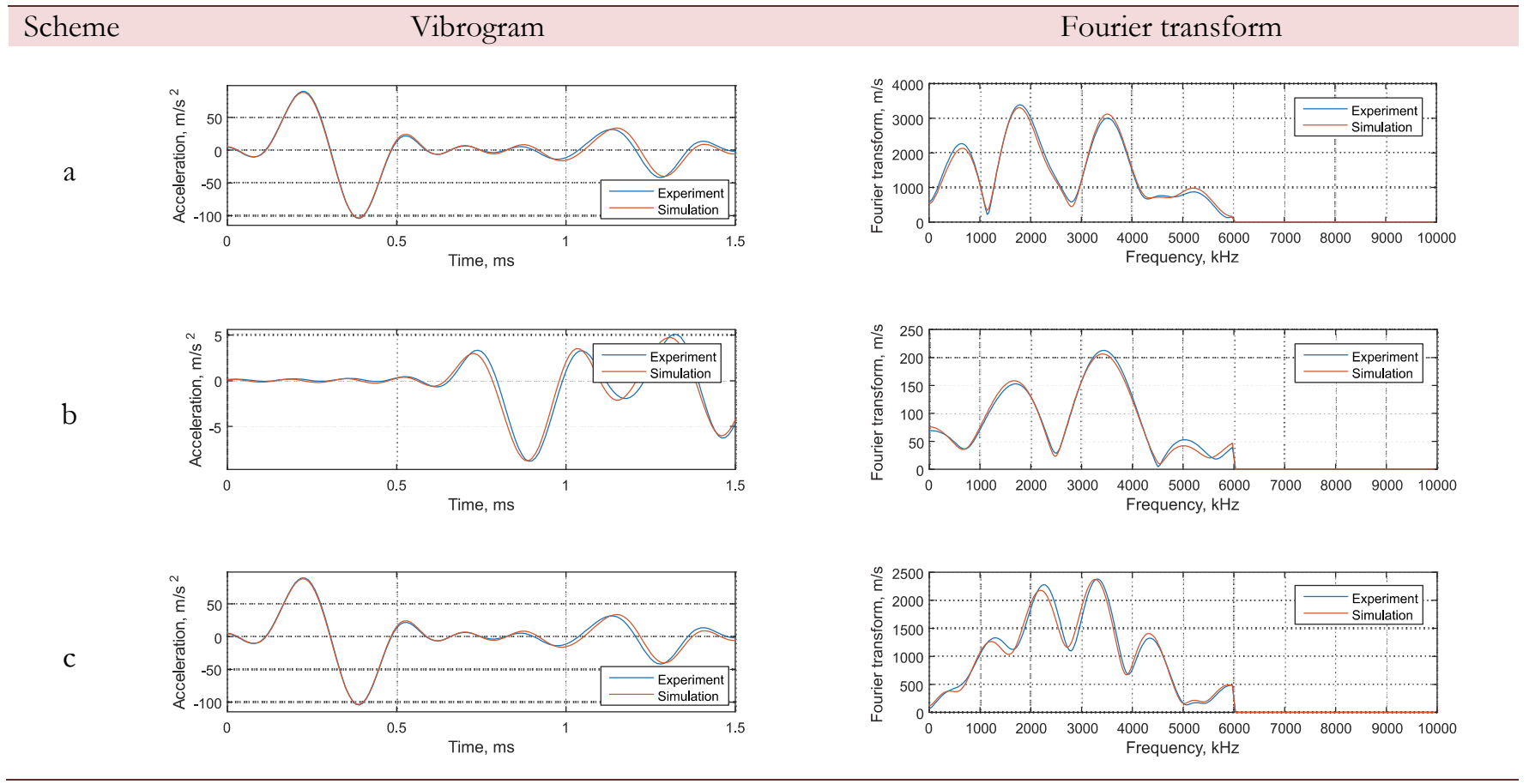

Figure 5: Vibrograms and corresponding Foutier transforms for three loading schemes.

\section{ANALYSIS OF CHANGES IN VIBROGRAMS CAUSED BY CRACK INITIATION}

$\mathrm{T}$

he proposed model (1)-(5), which passed the verification procedure, was used for numerical analysis of changes in the vibration parameters of the RC structure in the period of nucleation and propagation of cracks. The most probable places for crack formation in the structure are the connections of vertical columns with floor slabs and cross beams. In accordance with Fig. 6, we considered a fragment of the structure, for which we simulated the subsequent nucleation of cracks at the crossbar-to-column connections. Four crack nucleation stages were identified: the crack T1 appeared at the first stage, and cracks T2, T3 and T4 were successively formed at the next three stages. The numerical experiment consists in solving successively the problems of the dynamic deformation response of the structure to equal, locally applied impulse loads at all stages of crack nucleation. The examined structural fragment, the schemes of loading and registration of deformation response correspond to the scheme shown in Fig. 3b. To characterize the external force action, we used the parameters of the force impulse, determined experimentally at the stage of verification of the mathematical model.

As before, the implementation of the numerical experiment was based on the finite element method using the ANSYS software package. To simulate the crack, we excluded from the calculation one layer of finite elements for concrete adjacent to the face of the column, retaining yet the finite elements for the reinforcement to provide simulation of its integrity. As already mentioned, the evolution of crack formation was determined by a sequence of 4 stages: the first stage is associated with the formation of crack T1 followed by successive nucleation of cracks T2, T3, and T4 at the next three 
stages. The cracks were located in the cross sections of the cross beams. The depth of the crack was $150 \mathrm{~mm}$, the thickness of the undestroyed part of the crossbar was $100 \mathrm{~mm}$. The location of the prospective sensor for recording the dynamic deformation response is indicated by a red dot on the vertical column above the crack formation zone (point $\mathrm{S}$ in Fig. 6). It is evident that the cracks always locate in the region between the impact point and the sensor.

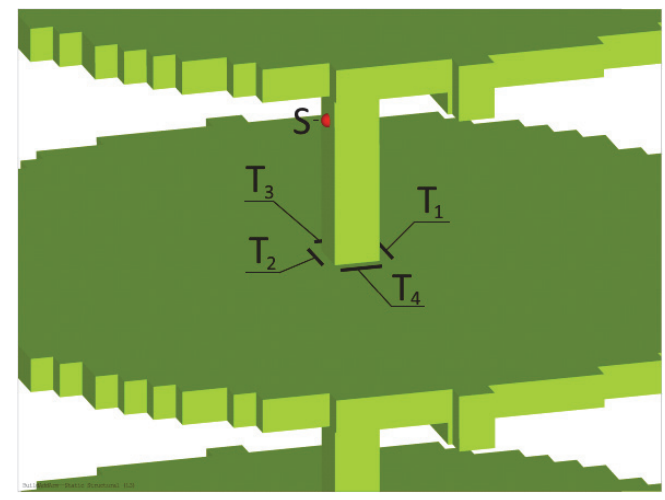

Figure 6: Location of cracks at the bottom of the column.

The results of the numerical simulation in the form of vibrograms and their Fourier images for point $\mathrm{S}$ are shown by red lines in the graphs in Fig. 7. A blue line shown in the same graphs for the sake of comparison corresponds to the experimental data obtained for the defect-free structure (Fig. 5, vibrogram b). These results demonstrate that a significant distortion of the vibration patterns and their Fourier images occurs at each stage after nucleation of a new crack.

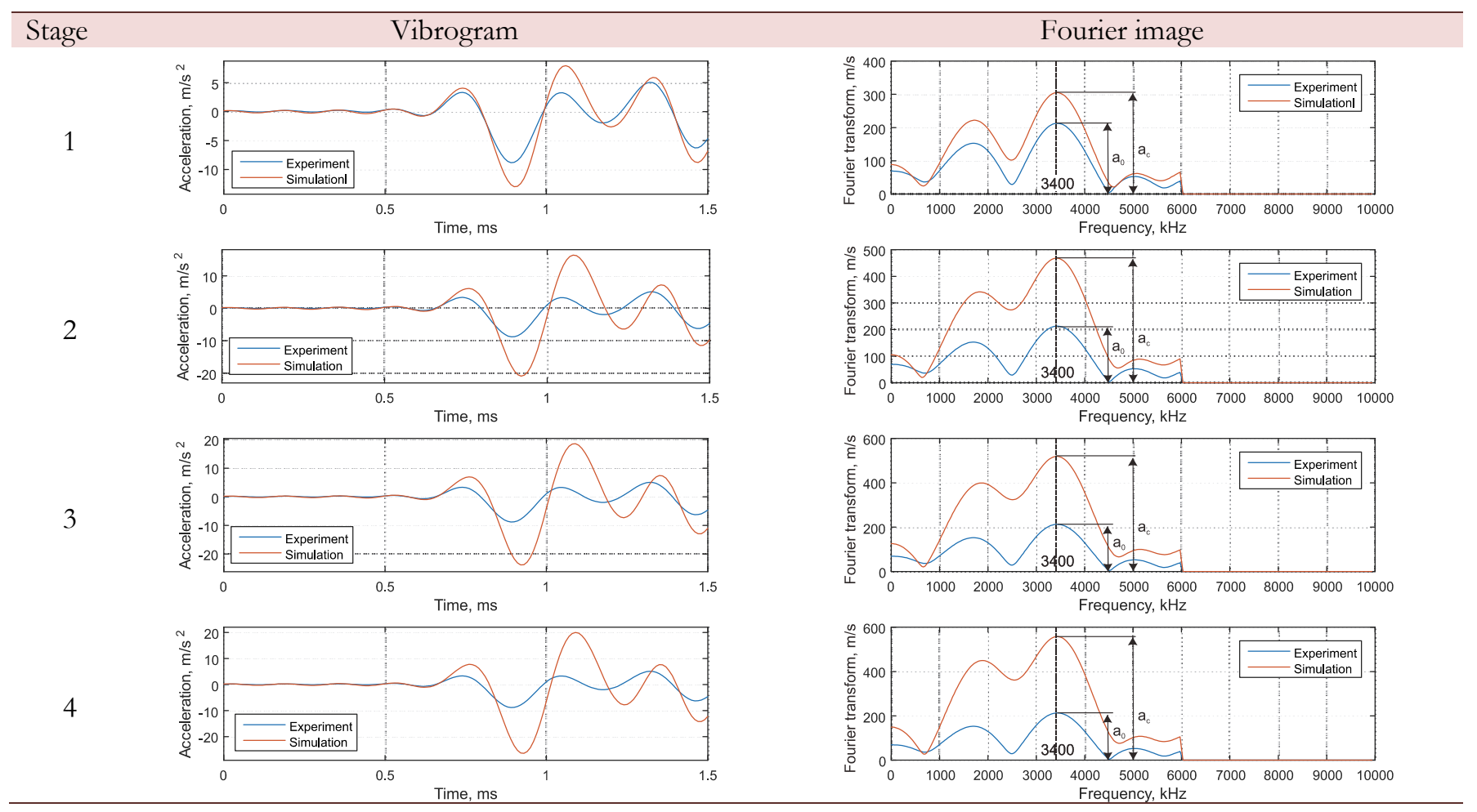

Figure 7: Changes in vibrograms at crack formation.

The most intense free vibrations of the structure are realized at frequencies around $3400 \mathrm{~Hz}$. A comparison of the amplitude value $a_{k}$ of the Fourier image at this frequency with the value $a_{0}$ obtained at the same frequency in the defectfree structure carries important information. The ratio of these values $K^{*}=a_{k} / a_{0}$ can be considered as an informative parameter that responds to the process of crack growth. Fig. 7 shows a variation in the criterion $K^{*}$ taken at the 
frequency of $3400 \mathrm{~Hz}$ (corresponding to the most intense vibrations) with the growth of damage in the structure. The first column corresponds to the defect-free structure, and the other columns - to the structures, in which the number of cracks increases. In this case, the value of the criterion increases markedly.

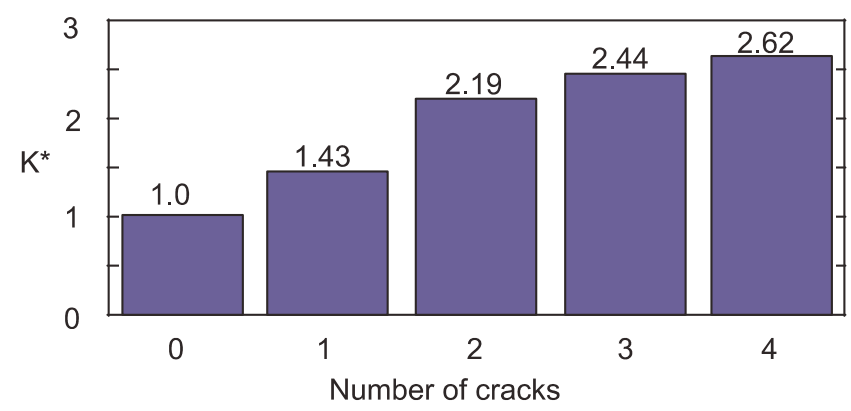

Figure 8: Criterion $K^{*}=a_{k} / a_{0}$ at different stages of cracking.

\section{CONCLUSION}

$\mathrm{I}$

$\mathrm{n}$ this work, verification of a mathematical model of dynamic processes in a reinforced concrete structure was carried out within the framework of viscoelasticity. A comparison of the experimental and calculated data has demonstrated that this model can be effectively used to describe with sufficient accuracy the propagation of vibration processes initiated by external, locally applied impact loads.

A series of numerical experiments performed on the basis of the mathematical model allowed us to investigate changes in the vibration parameters of the RC structure, in which the process of crack formation has started. As a parameter, characterizing the process of crack nucleation, we used the ratio of the amplitude values of the Fourier transform in the defect and defect-free structure obtained at the frequency of the most severe vibrations.

On the whole, the results of this study served to determine the substance of the desired algorithm for implementing the vibration diagnosis of reinforced concrete structures with the aim to control the process of crack formation.

\section{ACKNOWLEDGMENTS}

he research was performed at the Institute of Continuous Media Mechanics Ural Branch of Russian Academy of Science with the support of the Russian Science Foundation (project №14-29-00172).

\section{REFERENCES}

[1] Verma, S.K., Bhadauria, S.S. and Akhtar, S. (2013). Review of non destructive testing methods for condition monitoring of concrete structures, J. Constr. Eng, 834572. DOI: 10.1155/2013/834572.

[2] Fan, W. and Qiao, P. (2011). Vibration-based damage identification methods: a review and comparative study, Structural Health Monitoring. 10(1), pp. 83-111. DOI: 10.1177/1475921710365419.

[3] Stepinski, T., Uhl, T., and Staszewski, W. (2013). Advanced structural damage detection: from theory to engineering applications., John Wiley \& Sons. DOI:10.1002/9781118536148.

[4] Wang, L. and Chan, T.H.T. (2009). Review of vibration-based damage detection and condition assessment of bridge structures using structural health monitoring., Proc. of the second infrastructure theme postgraduate conference. Queensland University of Technology. Paper ID 26738. http://eprints.qut.edu.au/.

[5] Quaranta, G., Carbonu, B. and Lacarbonara, W. (2014). Damage detection by modal curvatures: numerical issues, J. Vibr. Contr. 22 (7) pp. 1913-1927. DOI: 10.1177/1077546314545528.

[6] Bykov, A.A., Matveenko, V.P., Serovaev, G.S., Shardakov, I.N. and Shestakov, A.P. (2015). Mathematical modeling of vibration processes in reinforced concrete structures for setting up crack initiation monitoring, Mech. Solids, 50 (2), pp. 160-170. DOI: 10.3103/S0025654415020053. 
[7] Raghavan A. and Cesnik C.E.S. (2007). Review of guided-wave structural health monitoring, Shock and Vibration Digest, 39 (2), pp. 91-114. DOI: 10.1177/0583102406075428.

[8] Liu, X.L., Jiang, Z.W. and Ji, L. (2013). Investigation on the design of piezoelectric actuator/sensor for damage detection in beam with lamb waves, Exp. Mech. 53 (3), pp. 485-492. DOI: 10.1007/s11340-012-9646-9.

[9] Song G., Gu H., Mo Y. L., Hsu T. T. C., Dhonde H. (2007). Concrete structural health monitoring using embedded piezoceramic transducers, Smart Mater. Struct. 16(4), pp. 959-968. DOI: 10.1088/0964-1726/16/4/003.

[10] Bykov, A.A., Matveenko, V.P., Shardakov, I.N. and Shestakov, A.P. (2017). Shock wave method for monitoring crack repair processes in reinforced concrete structures, Mech. Solids 52 (4), pp. 378-383. DOI: $10.3103 /$ S0025654417040033.

[11] Fröjd P. and Ulriksen P. (2016). Amplitude and phase measurements of continuous diffuse fields for structural health monitoring of concrete structures, NDT\&E International, 77, pp. 35-41. DOI: 0.1016/j.ndteint.2015.10.003.

[12] Xu, K., Deng, Q., Cai, L., Ho, S. and Song, G (2018). Damage detection of a concrete column subject to blast loads using embedded piezoceramic transducers, Sensors, 18 (5), 1377. DOI: 10.3390/s18051377. 